\title{
Functionalization and Area-Selective Deposition of Magnetic Carbon-Coated Iron Nanoparticles from Solution
}

\author{
Erika Widenkvist, ${ }^{1}$ Oscar Alm, ${ }^{1}$ Mats Boman, ${ }^{1}$ Ulf Jansson,, ${ }^{1}$ and Helena Grennberg ${ }^{2}$ \\ ${ }^{1}$ Department of Materials Chemistry, Uppsala University, Box 538, SE-752 21 Uppsala, Sweden \\ ${ }^{2}$ Department of Biochemistry and Organic Chemistry, Uppsala University, Box 576, 75123 Uppsala, Sweden
}

Correspondence should be addressed to Helena Grennberg, helena.grennberg@biorg.uu.se

Received 31 March 2011; Accepted 21 June 2011

Academic Editor: Quan Wang

Copyright (C) 2011 Erika Widenkvist et al. This is an open access article distributed under the Creative Commons Attribution License, which permits unrestricted use, distribution, and reproduction in any medium, provided the original work is properly cited.

\begin{abstract}
A route to area-selective deposition of carbon-coated iron nanoparticles, involving chemical modification of the surface of the particles, is described. Partial oxidative etching of the coating introduces carboxylic groups, which then are esterified. The functionalized particles can be selectively deposited on the $\mathrm{Si}$ areas of $\mathrm{Si} / \mathrm{SiO}_{2}$ substrates by a simple dipping procedure. Nanoparticles and nanoassemblies have been analyzed using SEM, TEM, and XPS.
\end{abstract}

\section{Introduction}

Materials composed of nanostructures are of great interest in the search for new functional materials. Magnetic iron nanoparticles are for example potential candidates for a variety of applications ranging from data storage to drug delivery [1-4]. To make use of the size-dependent properties, it is crucial to have means to individualize the nanoparticles in a controlled manner. Many applications also require a simple and reliable process for area-selective deposition of nanoparticles on a surface. In situ growth of nanostructures on the desired surface area often involves relatively high temperatures, which can be a major drawback when it comes to choice of substrate. Significant amounts of impurities in the as-grown material can also be problematic in this approach. Postfabrication assembly of the nanoparticles is a way to overcome these problems, and within this category, solution-based methods are of particular interest due to the scalability. Deposition from solution can be simple, of low-cost and relatively easy to scale up, provided the inherent problem of insolubility and aggregation of the nanostructures in solution is resolved. We have previously developed a solution-based area-selective deposition process for covalently functionalized multiwalled carbon nanotubes (MWCNTs) [5]. The nanotubes form stable solutions, and area-selective deposition on silicon/silicon oxide patterned surfaces was achieved for esterified nanotubes by a dip-rinsedry protocol.

In the present study, we have expanded the scope of our area-selective deposition protocol to also include magnetic carbon-coated Fe nanoparticles produced by laser-assisted chemical vapor deposition (LCVD). The particles consist of a metallic nucleus surrounded by a carbon shell containing both graphitic and amorphous carbon, thus the outer shell would be susceptible for the same type of chemistry as previously developed for carbon nanotubes [6-10]. Removing the amorphous outer layer and exposing the inner graphitic layer should render the functionalization and deposition process equally applicable to the coated iron nanoparticles.

\section{Results and Discussion}

The two-step scheme used to functionalize the carboncoated iron nanoparticles is presented in Figure 1. Due to the magnetic properties of the particles, the procedure developed for carbon nanotube functionalization was not directly transferable. In particular, use of magnetic stir bars for the mixing of reagents is inappropriate, and instead, boiling chips were employed to avoid local superheating. As these do not give as efficient mixing, longer reaction times and occasional sonication was employed. 


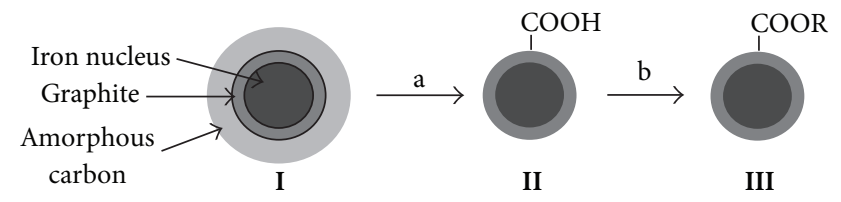

FIGURE 1: Two-step transformation of the as-synthesized core-shell iron nanoparticles with an amorphous and graphitic carbon coating (I) into ester functionalized core-shell nanoparticles (III). Step (a), treatment with aqueous $\mathrm{HNO}_{3}$, gives particles with a graphitic coating and carboxylic acid functional groups (II), and step (b), base and octyl bromide, transforms the acid groups into esters (III).

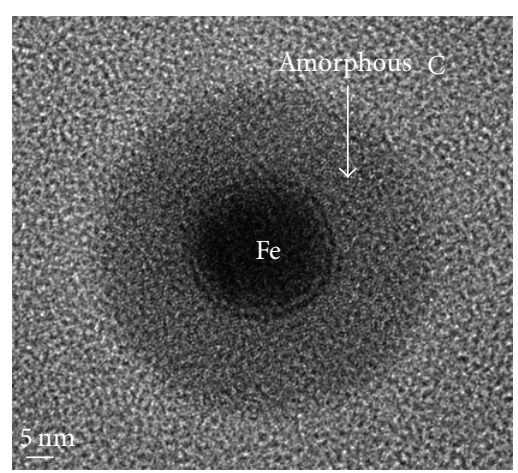

(a)

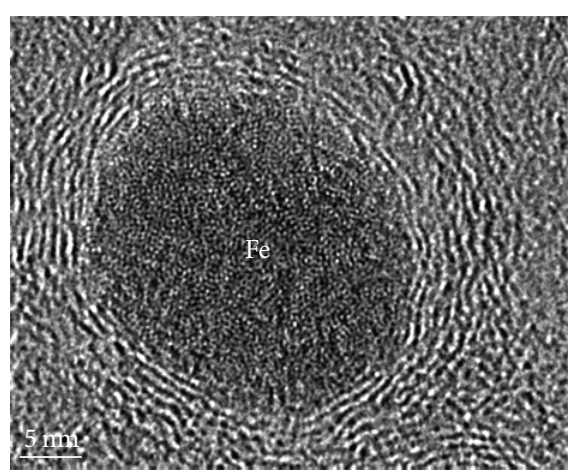

(b)

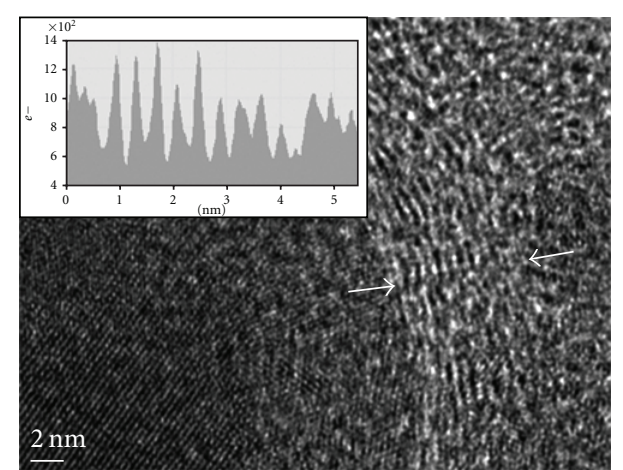

(c)

FIGURE 2: TEM of (a) as-produced core-shell nanoparticle (I), (b) an ester functionalized nanoparticle (III) (c), and The graphitic layer of III. Inset: interlayer spacing along the line between the arrows.

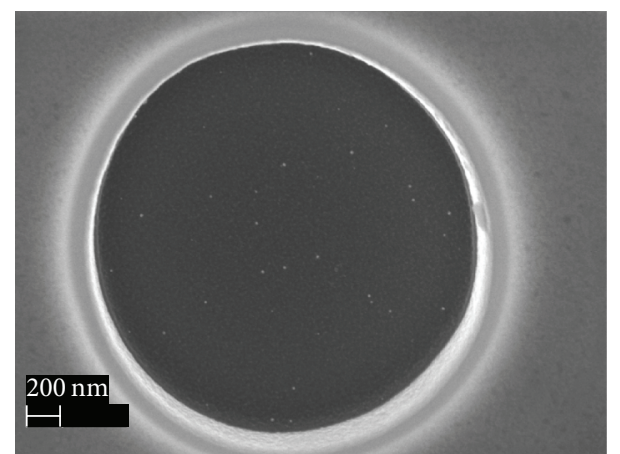

FIGURE 3: SEM-micrograph of a silicon/silicon oxide substrate after dip deposition of III. On the silicon area (black), particles are seen as small bright spots. The silicon oxide surface almost lack particles.

Separation of the functionalized nanoparticles from solvent and byproducts after the first reaction step was challenging. For MWCNTs, having a very high aspect ratio, filtration of quenched reaction solutions is usually sufficient to collect the product. In the case of our particles, both the dimensions and chemical properties are very similar to those of the carbon impurities. Thus, filtration would yield a mixture of desired and nondesired compounds, as would centrifugation. In order to overcome this problem, a magnetic-fieldassisted sedimentation procedure was used. The reaction flask was placed in an external magnetic field, causing the nanoparticles to aggregate close to the magnet [10]. The remaining dispersion, containing considerably less magnetic particles, could then be removed using a pipette. It should be noted that the current preparations were carried out using very small quantities of material, which in particular affect the yield of isolation procedures, as compared to the results when working on a larger scale. After the second step of the functionalization, the nanoparticles could be separated from the reaction mixture by conventional extraction. In addition, magnetic-field-assisted sedimentation was used to wash the particles and also to increase the concentration of the stock solution.

The magnetic properties of the as-produced particles I are preserved in III, which is as expected from a purification scheme involving capture of all magnetic material. The properties were also confirmed from magnetic measurements on a chloroform solution of III. Further characterization of the functionalized particles in solution has proven to be difficult. Dynamic light scattering measurements has so far given less conclusive results; this is most probably an effect of remaining amorphous carbon impurities, much larger than the nanoparticles, disturbing the measurement. In addition, analysis of a dispersion of $\mathbf{I}$ was not possible using this method, due to large aggregates in the sample.

TEM analysis of III (Figure 2) showed a successful removal of amorphous carbon during the first step of the functionalization, leaving the graphite layer intact. The interlayer spacing of the remaining shell, seen in Figure 2(c), was measured to be $3.6 \AA$, which is close of that of MWCNTs and graphitic carbon (3.4 $\AA$ ) [11]. The esterified nanoparticles are, by the added alkyl chains, nonpolar and hydrophobic enough to be extracted from water to chloroform, indicating a preference for a nonpolar environment that 


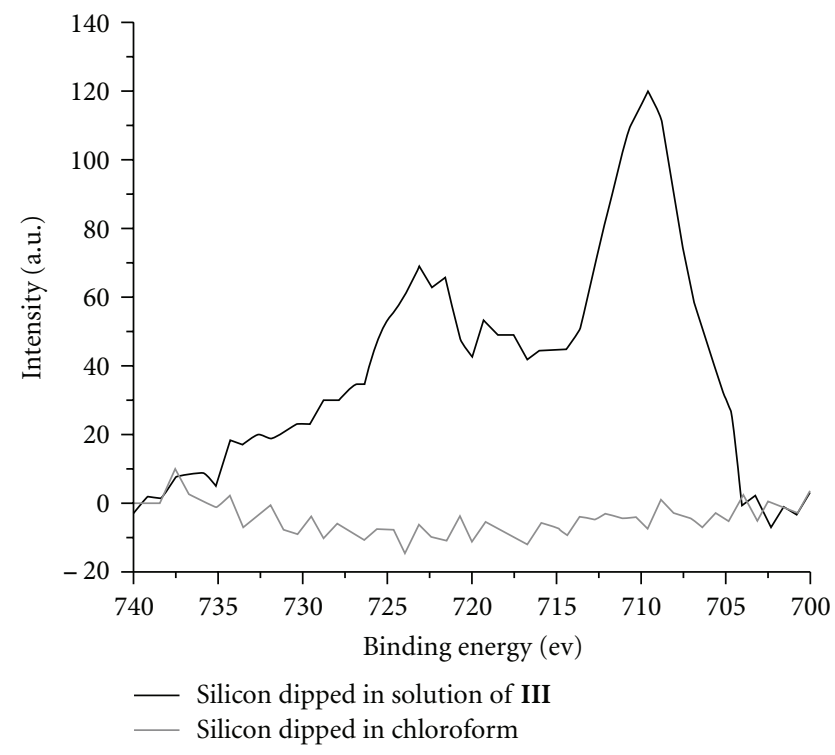

(a)

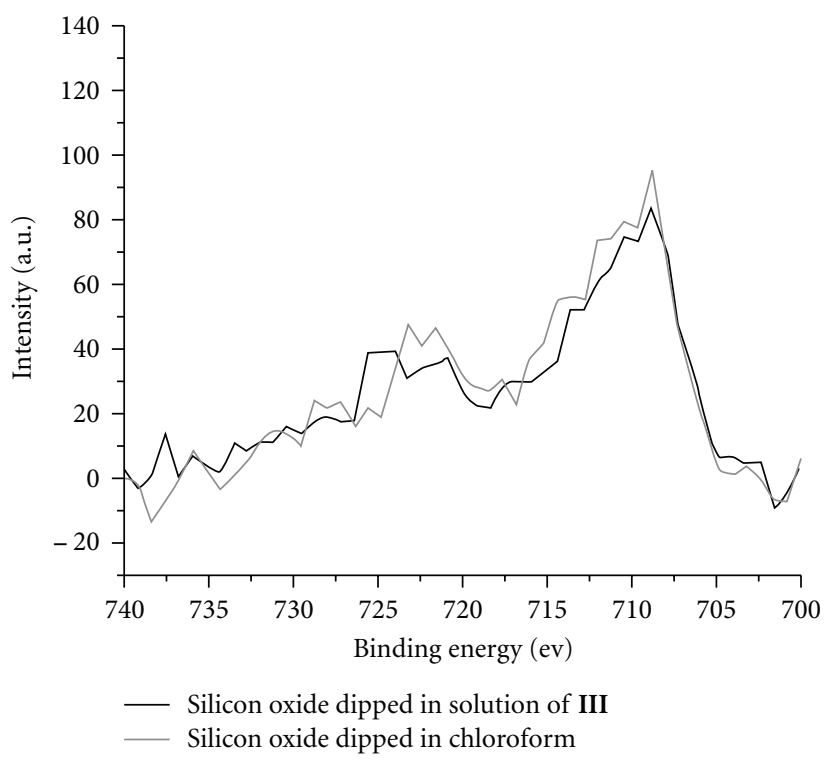

(b)

FIGURE 4: The XPS spectra of the Fe2p peak for (a) a silicon substrate treatment with a chloroform solution of III and a reference substrate treated with pure chloroform and (b) a silicon oxide substrate after treatment with a chloroform solution of III and a reference substrate treated with pure chloroform.

is a prerequisite for area-selective deposition on patterned $\mathrm{Si} / \mathrm{SiO}_{2}$ substrates.

After HF-etching, the Si surface is hydrogen-terminated and highly hydrophobic $[12,13]$. In contrast, the $\mathrm{SiO}_{2}$ surface is terminated by $\mathrm{Si}-\mathrm{OH}$ groups and consequently very hydrophilic. By dipping the substrate into a solution III, area-selective adsorption should be possible due to hydrophobic interactions between the $\mathrm{Si}-\mathrm{H}$ surface and the particles, in analogy with the MWCNT results. The magnetic properties made SEM imaging more challenging, and for dimensionality reasons, the particles are less easily distinguishable than are MWCNTs. Still, SEM of patterned $\mathrm{Si}-\mathrm{H} / \mathrm{Si}-\mathrm{OH}$ substrates dipped into solutions of III provided clear evidence for area-selective adsorption, with the nanoparticles almost exclusively adsorbed at the hydrophobic hydrogen-terminated Si areas (Figure 3).

The SEM observations were confirmed by XPS. Comparison of hydrogen-terminated Si substrates treated with solutions of III or with pure solvent confirmed the presence of Fe on the surface on samples treated with III (Figure 4(a)). Silicon oxide substrates treated simultaneously displayed no differences as, somewhat surprisingly; the same amount of $\mathrm{Fe}$ was detected on reference samples not exposed to III (Figure 4(b)). The origin of the Fe intensity from the silicon oxide substrates is most probably due to impurities in the as-received substrates. Taken together, our results clearly show that area-selective deposition of functionalized nanoparticles is obtained. The esterification is essential, neither as-produced (I) nor carboxylated (II) particles can be deposited in this manner.

\section{Conclusion}

We have successfully developed a protocol for covalent functionalization of LCVD synthesized carbon-coated core- shell iron nanoparticles, and demonstrated that the functionalized particles can be adsorbed area-selectively onto silicon/silicon oxide surfaces from solution. The protocol gives stable solutions of carbon-coated metal nanoparticles in organic solvents and further process steps such as centrifugation or chromatography can be applied to obtain sizeseparated fractions. Furthermore, the observed selectivity of the functionalized nanoparticles to hydrophobic surfaces can be used for scalable fabrication of designed magnetic structures controlled by the surface chemistry.

\section{Experimental}

4.1. General. All chemicals used were obtained from commercial sources and were of analytical grade if not otherwise specified. Carbon coated Fe nanoparticles (log-normal size distributed with a total geometric mean diameter of $\sim 20 \mathrm{~nm}$ ( $\sigma \approx 1.5 \mathrm{~nm}$ ) with an Fe core of $\sim 8 \mathrm{~nm}$ in diameter) were produced by Laser Assisted Chemical Vapor Deposition (LCVD) by procedures described elsewhere $[13,14]$.

4.2. Functionalization. A two-step scheme was used to functionalize the carbon-covered iron nanoparticles (Figure 1), inspired by previous work with carbon nanotubes [6]. Due to the magnetic properties of the Fe nanoparticles, conventional stir bars should be avoided in all steps involving solutions of the particles in order to avoid loss of material due to interaction with the stir bar.

The LCVD-synthesized material I was mechanically removed from the silicon support substrate in a roundbottom reaction flask in the presence of deionized water. The resulting particle-water mixture was briefly sonicated to disperse the particles and concentrated $\mathrm{HNO}_{3}(0.15 \mathrm{~mL} / \mathrm{mL}$ of I-containing dispersion to obtain a $\mathrm{HNO}_{3}$ concentration of $2 \mathrm{M}$ ) and a boiling chip were added. After a brief 
sonication, the flask was equipped with a reflux condenser and the mixture was heated at $95^{\circ} \mathrm{C}$ in an oil-bath for $5 \mathrm{~h}$. Aqueous $\mathrm{NaOH}(1 \mathrm{M})$ was added to neutralize the acid, and then separation of the oxidized nanoparticles from the reaction solution was achieved by magnetic-field-assisted sedimentation: A neodymium magnet was placed below the reaction vessel for at least $30 \mathrm{~min}$ at $\mathrm{RT}$. This attracted the magnetic particles, seen as black/brown aggregates, to the lower part of the vessel and most of the liquid could be removed using a pipette. The product (II) obtained after cleaning of the particles by addition of water, swirling of the vessel, magnetically assisted sedimentation, and removal of most of the wash solvent was used without further treatment in the next step.

In the second step, a boiling chip, aqueous $\mathrm{NaOH}$ ( $40 \mathrm{~mL}, 1 \mathrm{mM}$ ), hexadecyl-trimethyl-ammonium bromide $\left(\mathrm{C}_{19} \mathrm{H}_{42} \mathrm{BrN}, 1 \mathrm{mg}\right)$ and octyl bromide $\left(\mathrm{C}_{8} \mathrm{H}_{17} \mathrm{Br}, 1 \mathrm{~mL}\right)$ was added to a reaction flask containing the product from the first step. After a brief sonication, the flask was equipped with a reflux condenser and the dispersion was refluxed for $3 \mathrm{~h}$. After allowing the reaction mixture to cool to RT and a brief sonication, $\mathrm{CHCl}_{3}$ was added in order to extract the nonpolar compounds. The clear organic layer was collected and washed with deionized water in a separation funnel. The volatile organics (chloroform and excess octyl bromide) was removed by vacuum distillation, and the resulting crude ester functionalized nanoparticles were dissolved in $\mathrm{CHCl}_{3}$. The solution was used as stock solution for the deposition experiments. Reference dispersions of the nonfunctionalized nanoparticles were prepared by mechanical removal of the as-produced particles from the synthesis substrate in the presence of $\mathrm{CHCl}_{3}$ followed by a brief sonication.

Samples from the stock solutions of III as well as the reference dispersion of I were characterized by transmission electron microscopy (TEM) using a FEI Tecnai F30 ST and a JEOL 2000 FXII microscope. Samples were prepared by placing droplets on copper grids with a lacey carbon support film and allowing the solvent to evaporate.

4.3. Substrate Pretreatment. The substrates used in the experiments were n-type (100) silicon and amorphous silicon oxide, both obtained from commercial sources, as well as silicon substrates with a silicon oxide pattern prepared by conventional lithographic techniques. Prior to treatment with solutions of III or controls, the substrates were degreased by a sequence of $5 \mathrm{~min}$ sonication, using (1) trichloroethylene and (2) acetone, followed by rinsing with ethanol and drying in a flow of $\mathrm{N}_{2}$. Hydrogen-terminated silicon surfaces were prepared by etching away the native oxide on the surface in $2 \%$ HF solution for $15 \mathrm{~s}$, followed by rinsing with deionized water and drying in a flow of $\mathrm{N}_{2}$.

4.4. Deposition. The substrates were immersed vertically in a chloroform solution of III followed by a rinse using pure chloroform and drying in air. The deposited samples were characterized by means of scanning electron microscopy (SEM) using a LEO 1550 microscope equipped with an Inlens detector and a field-emission gun. The amount of iron present on the samples was investigated by XPS using a PHI
Quantum 2000 instrument. The analysis was performed with a $15^{\circ}$ exit angle to increase the intensity originating from the particles on the surface.

\section{Acknowledgments}

This work has received financial support from the Swedish Research Council. Professor Peter Svedlindh and Claes-Henrik Andersson are acknowledged for assistance with magnetic measurements and functionalization advice, respectively.

\section{References}

[1] G. Cao, Nanostructures and Nanomaterials: Synthesis, Properties and Applications, Marcel Dekker, New York, NY, USA, 1997.

[2] S. P. Gubin and Y. A. Koksharov, "Preparation, structure, and properties of magnetic materials based on co-containing nanoparticles," Inorganic Materials, vol. 38, no. 11, pp. 10851099, 2002.

[3] A. H. Lu, E. L. Salabas, and F. Schüth, "Magnetic nanoparticles: synthesis, protection, functionalization, and application," Angewandte Chemie International Edition, vol. 46, no. 8, pp. 1222-1244, 2007.

[4] X. Yang, X. Zhang, Y. Ma, Y. Huang, Y. Wang, and Y. Chen, "Superparamagnetic graphene oxide- $\mathrm{Fe}_{3} \mathrm{O}_{4}$ nanoparticles hybrid for controlled targeted drug carriers," Journal of Materials Chemistry, vol. 19, no. 18, pp. 2710-2714, 2009.

[5] E. Widenkvist, J. Li, U. Jansson, and H. Grennberg, "Selected area deposition of multiwalled carbon nanotubes from solution," Carbon, vol. 45, no. 14, pp. 2732-2736, 2007.

[6] J. Li and H. Grennberg, "Microwave-assisted covalent sidewall functionalization of multiwalled carbon nanotubes," Chemistry - A European Journal, vol. 12, no. 14, pp. 3869-3875, 2006.

[7] A. S. Rettenbacher, B. Elliott, J. S. Hudson, A. Amirkhanian, and L. Echegoyen, "Preparation and functionalization of multilayer fullerenes (carbon nano-onions)," Chemistry - A European Journal, vol. 12, no. 2, pp. 376-387, 2005.

[8] S. Banerjee, T. Hemraj-Benny, and S. S. Wong, "Covalent surface chemistry of single-walled carbon nanotubes," Advanced Materials, vol. 17, no. 1, pp. 17-29, 2005.

[9] K. Balasubramanian and M. Burghard, "Chemically functionalized carbon nanotubes," Small, vol. 1, no. 2, pp. 180-192, 2005.

[10] R. N. Grass, E. K. Athanassiou, and W. J. Stark, "Covalently functionalized cobalt nanoparticles as a platform for magnetic separations in organic synthesis," Angewandte Chemie International Edition, vol. 46, no. 26, pp. 4909-4912, 2007.

[11] P. M. Ajayan, "Nanotubes from carbon," Chemical Reviews, vol. 99, pp. 1797-1799, 1999.

[12] D. B. Fenner, D. K. Biegelsen, and R. D. Bringans, "Silicon surface passivation by hydrogen termination: a comparative study of preparation methods," Journal of Applied Physics, vol. 66, no. 1, pp. 419-424, 1989.

[13] P. Dumas, Y. J. Chabal, and P. Jakob, "Morphology of hydrogen-terminated $\mathrm{Si}(111)$ and $\mathrm{Si}(100)$ surfaces upon etching in HF and buffered-HF solutions," Surface Science, vol. 269-270, no. C, pp. 867-878, 1992.

[14] K. Elihn, F. Otten, M. Boman et al., "Size distributions and synthesis of nanoparticles by photolytic dissociation of ferrocene," Applied Physics A, vol. 72, no. 1, pp. 29-34, 2001. 

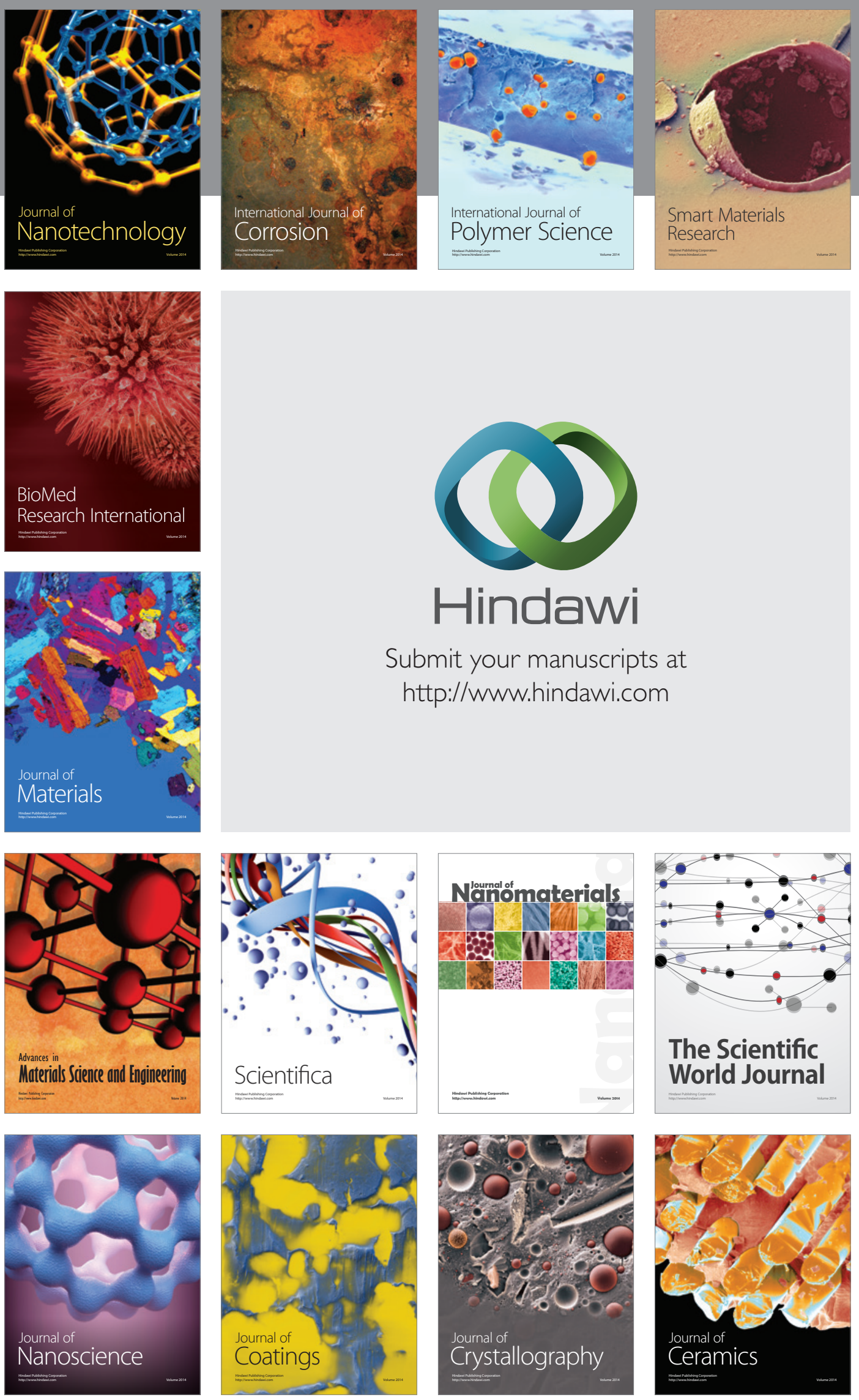

The Scientific World Journal

Submit your manuscripts at

http://www.hindawi.com

\section{World Journal}

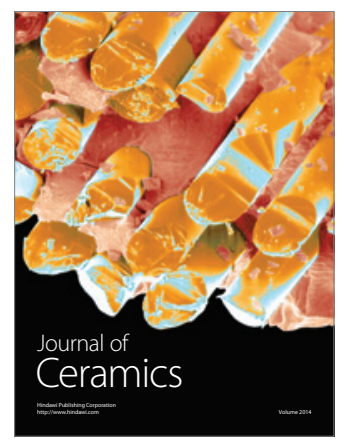

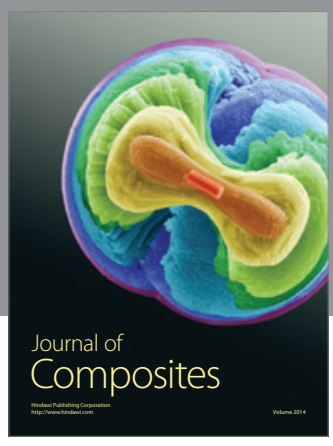
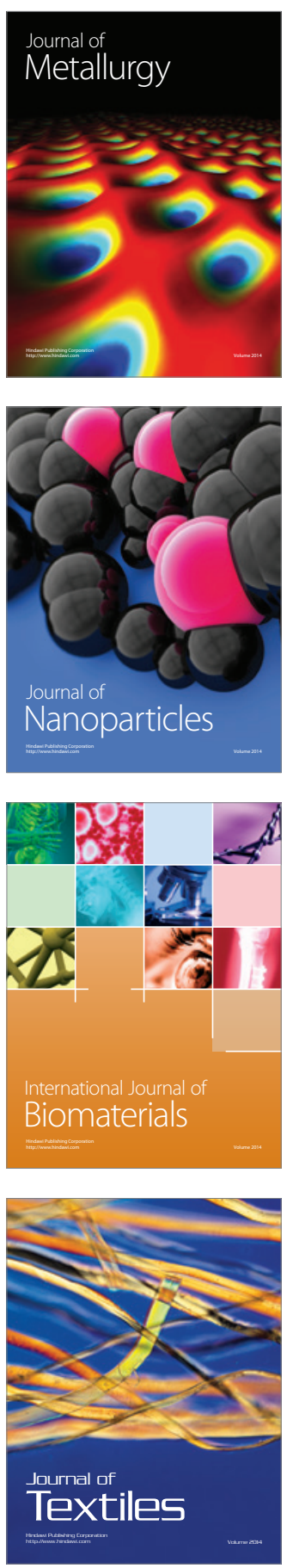